\title{
Does Inulin Ingestion Reduce Visceral Fat Adiposity? Mini Review
}

\author{
Laila A AlBishi* \\ Department of Pediatric, University of Tabuk, Saudi Arabia
}

Received: March 01, 2018; Published: March 20, 2018

*Corresponding author: Laila A AlBishi, Department of Pediatric, Assistant Professor Pediatric Endocrinologist, Pediatric department, Faculty of Medicine, University of Tabuk, Saudi Arabia, Tel: 966 500711130; Email: lalbishi@ut.edu.sa

\begin{abstract}
Abbreviations: VT: Visceral Fat; VAT: Visceral Adipose Tissue; IR: Insulin Resistance; MetS: Metabolic Syndrome; T2DM: Type 2 Diabetes Mellitus; BMI: Body Mass Index; LDL: Low Density Lipoprotein; SCFA: Short Chain Fatty Acids; ITF: Inulin-Type Fructan; FOS: Fructo Oligopolysacchrides; PYY: Peptide YY; RCT: Randomized Clinical Trials; GLP-1: Glucagon like Peptide-1; HDL: High Density Lipoprotein; BW: Body Weight
\end{abstract}

\section{Introduction}

Undoubtedly, obesity has become a global public health problem particularly when looking at the obesity-related diseases. Over the past 20 years, studies explored greatly the role of fat tissue in obesity pathogenesis suggesting that the functional differences between visceral fat adiposity (VFA) and subcutaneous fat adiposity (SFA) impacted the appearance of many obesity-related diseases. Therefore, increased knowledge about VFA and SFA could promote the development of more effective therapies against several obesity- related problems. The role of modulating gut microbiota with fiberdiet (prebiotics) in attempt to reduce VFA is nowadays an era of interest to treat obesity. Prebiotics constitute a selective stimulation of growth/activity of certain microbial species in the gut microbiota that offer health benefits to the host [1]. Consequently, its use as a dietary intervention provides a novel therapeutics in obesity and cardio-metabolic disease risk [2]. These mini-review summaries the current evidence linking a specific dietary-fiber called inulin to the reduction of VFA.

- Decrease in gene expression of hepatic enzymes involved in lipid synthesis

- Increase of muscle lipoprotein lipase enzyme activity

- Enhancing the production of short-chain fatty acids

- Altered production of polyamines which increases the production of satiogenic peptide

- Altered blood glucose and insulinemia

- Increase of fecal excretion of bile salts and cholesterol

- Increase of the Bifidobacterium population.

Figure 1: Mechanisms of insulin-type fructans in decreasing lipid profile.

\section{Inulin and its Health Benefits}

Inulin is a chicory root fiber that also found in onions, garlic, wheat, artichokes and bananas. It is a polymer of fructose monomers that is indigestible but soluble fiber. The estimated daily consumption ranges between 3-11 grams in Europe and 1-4 grams in the United States [3]. Studies demonstrated several physiological functions of inulin such as modulation of lipid metabolism in the liver and muscle. Reis et al. explained the main mechanisms 
of inulin-type fructans in the improvement of lipid profile, thus reducing the cardiovascular risk [4] (Figure 1). As the diet considered a strong contributor to the structural microbial changes [5], a prebiotics such as oligofructose (a subgroup of inulin) may affect the structural composition of the intestinal microbiota following high-fat diet feeding, thus improving the parameters of metabolic inflammation [6].

Interestingly, Cani [6,7] demonstrated that oligofructose consumption resulted in increased satiety and accompanied by a decrease in total energy intake in adults. This is explained by the presence of short chain fatty acids (SCFA) and their ability to increase appetite suppressing hormones such as glucagon-like peptide 1 (GLP-1) [7]. A large intestinal microflora fermentation facilitated a reduction in serum cholesterol and low density lipoprotein (LDL) levels 'important risk for coronary heart disease', [8] consequently, inulin found to exist a role in the stimulation of bifid bacteria growth "probiotics" [9].

\section{The link Between Intestinal Mirbiota and Visceral Fat Deposition}

VF is a type of adipose tissue which has an important implication for metabolic health [10]. Interestingly, VF is highly heritable and showed significant associations with the fecal microbiota According to Beaumont et al, studies linked increased abdominal adiposities using body fat distribution measures such as waist/ hip ratio, to fecal microbiome profiles in which specific probiotic consumption reported to lower VF. The authors suggested that VF mass plays a major role in the components of central adiposity or 'cardio-metabolic disease risk' [11]. More importantly, the presence or absence of a microbial flora regulates insulin sensitivity and cholesterol metabolism [12]. According to Tilg and Kaser, evidence indicated that type 2 diabetes mellitus (T2DM) negatively affect the structural composition of the microbiota (Bacteroidetes) which if decreased, the metabolic endotoxemia and systemic inflammatory markers might improve in diabetic mice [13]. Furthermore, microbiota produces enzymes that degrade ingested polysaccharides, therefore promoting the absorption of nutrients particularly carbohydrates, leading to increased liver lipogenesis, hepatic IR, and hyperinsulinemia.

Authors added that various dietary components such as wheat fiber, inulin, or starch with high amylase content improve glucose absorption, decrease insulin secretion, increase the incretin GLP-1 level, and increase SCFA production and absorption. In hepatic steatosis 'a health-related risk of obesity and T2DM', a proton magnetic resonance spectroscopy used to measure intrahepatocellular lipids and intramyocellular lipids showed a close correlation between body adiposity and hepatic steatosis [14]. Importantly, by giving Agave inulin (inulin type with different chemical structure and botanical origin) to healthy adults in a randomized dose of $0,5.0$, or $7.5 \mathrm{~g} /$ day, there was a positive correlation between agave inulin intake and Bifid bacterium which is a promising shift of the gastrointestinal microbiota composition and activity in healthy adults [15].

\section{Inulin and Visceral Fat Reduction}

In terms of body weight (BW) and fat mass reduction Abrams et al. observed a significant BMI reduction and less increase in fat mass for adolescents who received 8 grams oligofructose-enriched inulin daily for one year and this effect maintained in the following year [16]. A study by Dewulf et al. evaluated the contribution of the gut microbiota to the modulated host metabolism by dietary inulintype fructans (ITF) prebiotics in obese women and found that ITF decreased the fat mass, serum lipopolysaccharides, and plasma lactate and phospha tidylcholine levels [17]. A recent comparison between the effects of fructooligosaccharides (FOS) and highfat "western" diet with or without $2.5 \%$ FOS supplementation ad libitum on the development of obesity in rats showed that BW and percent body fat were lower FOS group while the weight of the visceral adipose tissue, and the weight and triglyceride content of the liver were significantly lower in the high-fat + FOS group.

Moreover, fecal excretion of lipids markedly potentiated by FOS consumption thus indicated the suppression effect of dietary FOS to the high-fat diet-induced body fat accumulation, and intestinal absorption of dietary fat [18]. Another comparison between nonfermentable ( $10 \%$ cellulose) and fermentable inulin, the results showed that the short-term inulin feeding in mice positively impacted the lipid metabolism [19]. On clinical grounds, inulin demonstrated a significant role in health-protective effects and disease-reversal benefits particularly with high level of intake. Data showed a positive impact on cardiovascular health disease, hypertension, diabetes, obesity, and certain gastrointestinal diseases. Additionally, high fiber intake improves serum lipoprotein values, lowers hypertension, improves blood glucose control for diabetic patients, and leads to weight loss [20].

Rebello et al. [21] examined the effects of a gastrointestinal microbiome modulator containing inulin on metabolic parameters, fecal markers of gut microbiota, and satiety where the results confirmed an improvement in glucose tolerance and satiety. Johnston et al. [22] demonstrated better insulin sensitivity with chronic consumption of resistant starch in individuals with MetS though a non-significant effect observed on BW, fat storage in muscle, liver or visceral depots. Additionally, there was no change on vascular function or markers of inflammation Fernandes, Vogt \& Wolever compared markers of colonic fermentation after acute inulin ingestion in individuals with normal $(<40 \mathrm{pmol} / \mathrm{l})$ and high ( $\geq 40 \mathrm{pmol} / \mathrm{l})$ plasma insulin. The result supported that inulin increases short-term markers for colonic fermentation, thus produced SCFAs, acetate, propionate and butyrate, which might protect from T2DM by reducing serum free-fatty acids [23]. There is evidence that inulin-type fructans increases the GLP-1 level in the proximal colon that has a role in improving glucose tolerance, glucose-stimulated insulin secretion, insulin-sensitive hepatic glucose production, and thus reducing weight gain.

Furthermore, OFS could modulate gastrointestinal peptides such as peptide YY (PYY) and ghrelin that involved in the control of food intake. Data in humans reported the control of satiety 
facilitated by FOS as well as triglyceridemia, and steatohepatitis [24]. In male lean and obese rats, Parnell \& Reimer evaluated the dose-dependent effects of inulin and oligofructose on gut satiety hormones, energy expenditure, gastric emptying and gut microbiota by giving randomized fiber intake of 0 to $20 \%$. The authors concluded that bacteroides and total bacteria correlated positively with ghrelin and negatively with insulin demonstrating that prebiotics action of food intake, satiety hormones and alterations in gut microbiota are regulated in a dose-dependent manner which could have therapeutic potential for obesity [25]. In a similar context but plateau result, a dose of daily 10 grams inulin for 12 weeks duration showed no significant effect on serum glucose and lipid levels in T2DM patients which raised important questions regarding the optimal dose of inulin as dose-response and also the optimal dosage that is not studied in humans [26].

Kim et al conducted a small randomized clinical trials (RCT) in medical students' evaluating the effects of fructooligosaccharides (FOS) supplements on BW and body composition, blood lipid profiles, and Ca status. The results demonstrated a significant decrease in BMI, body fat mass, percent body fat and VF while skeletal muscle mass increased. Also there was a decrease in serum total cholesterol, LDL-cholesterol and triglycerides level while HDL-cholesterol calcium related biomarkers increased. Authors suggested that habitual FOS consumption considered as effective for weight reduction, improving lipid profiles, and calcium status [27]. Guess et al. [28] concluded that inulin may reduce the risk of diabetes by promoting weight loss and decreasing intrahepatocellular and intramyocellular lipid in subjects with prediabetes independent of weight loss [28]. A recent systematic review by Liu et al. [29] assessed the effects of ITF on human lipid profiles and glucose homeostasis complicated by dyslipidemia, overweight or obesity, and T2DM.

The authors found that the ITF consumption associated LDL reduction across all study populations, however, improvements of HDL and glucose control demonstrated only in the T2DM subgroup. Thus, additional, well-powered, long-term, RCTs are required for a definitive conclusion. Overall, ITF supplementation may provide a novel direction for improving the lipid profile and glucose metabolism [29]. A further recent animal study demonstrated that the additive effect of enriched-diet containing both lactalbumin and inulin decreased energy intake, supra-additively decreased BW, fat, and lean mass, in addition to decreased respiratory quotient and plasma leptin concentrations [30].

\section{Conclusion}

The review supported short-term significant beneficial effects of inulin on body weight with particular attention to the VF accumulation and its consequences though most data demonstrated obvious positive effects on lipid profile. Some studies focused on a high dose to yield an optimal dose-response effect. On the other hand the lack of standard safety guidelines on oral administration of inulin as prebiotics agents in human necessitates a careful consumption. Additionally, further long-term studies are essential to increase the understanding of the complex efficacy between ingested inulin and cardio-metabolic risks.

\section{References}

1. Roberfroid M, Gibson GR, Hoyles L, McCartney AL, Rastall R, et al. (2010) Prebiotic effects: metabolic and health benefits. Br J Nutr 104 (2): 1-63.

2. Vinjé Sarah, Stroes Erik, Nieuwdorp Max, Hazen L Stan (2014) The gut microbiome as novel cardio-metabolic target: the time has come. Eur Heart J 35(14): 883-887.

3. Moshfegh AJ, Friday JE, Goldman JP, Ahuja JK. (1999) Presence of inulin and oligofructose in the diets of Americans. The Journal of Nutrition 129(7): 1407- 1411.

4. Reis SA, Conceição LL, Rosa DD, Dias MM1, Peluzio Mdo C (2014) Mechanisms used by inulin-type fructans to improve the lipid profile. Nutr Hosp 31(2): 528-534.

5. Zhang C, Zhang M, Wang S, Han R, Cao Y, et al. (2010) Interactions between gut microbiota, host genetics and diet relevant to development of metabolic syndromes in mice. ISME J 4(2): 232-241.

6. Cani PD, Neyrinck AM, Fava F, Knauf C, Burcelin RG, et al. (2007) Selective increases of bifidobacteria in gut microflora improve highfat-diet-induced diabetes in mice through a mechanism associated with endotoxaemia. Diabetologia 50(11): 2374-2383.

7. Cani PD, Joly E, Horsmans Y, Delzenne NM (2006) Oligofructose promotes satiety in healthy human: a pilot study. Eur J Clin Nutr 60(5): 567-572.

8. Amaral L, Morgan D, Stephen AM, Whiting S (1992) Effect of Propionate on Lipid-Metabolism in Healthy-Human Subjects. FASEB J 6(5): 1655.

9. Gibson GR, Beatty ER, Wang X, Cummings JH (1995) Selective stimulation of bifidobacteria in the human colon by oligofructose and inulin. Gastroenterology 108(4): 975-82.

10. Despres JP (2012) Body fat distribution and risk of cardiovascular disease: an update .Circulation 126: 1301-1313.

11. Michelle Beaumont, Julia K Goodrich, Matthew A Jackson, Idil Yet, Emily R Davenport, et al. (2016). Heritable components of the human fecal microbiome are associated with visceral fat. Genome Biol 17: 189.

12. Rabot S, Membrez M, Bruneau A, Gérard P, Harach T, et al. (2010) Germ-free C57BL/6J mice are resistant to high-fat-diet-induced insulin resistance and have altered cholesterol metabolism. FASEB J 24(12): 4948-459.

13. Tilg Herbert, Kaser Arthur (2011) Gut microbiome, obesity, and metabolic dysfunction. J Clin Invest 121 (6): 2126-2132.

14. Thomas EL, Hamilton G, Patel N, O'Dwyer R, Doré CJ, et al. (2005) Hepatic triglyceride content and its relation to body adiposity: a magnetic resonance imaging and proton magnetic resonance spectroscopy study. Gut 54(1):122-127.

15. Holscher HD, Bauer LL, Gourineni V, Pelkman CL, Fahey GC, et al. (2015) Agave Inulin Supplementation Affects the Fecal Microbiota of Healthy Adults Participating in a Randomized, Double-Blind, Placebo-Controlled, Crossover Trial. J Nutr 145(9): 2025-2032.

16. Abrams SA, Griffin IJ, Hawthorne KM, Ellis KJ (2007) Effect of prebiotic supplementation and calcium intake on body mass index. J Pediatr 151(3): 293-298.

17. Evelyne M Dewulf, Patrice D Cani, Sandrine P Claus, Susana Fuentes, Philippe GB Puylaert, et al. (2012) Insight into the prebiotic concept: Lessons from an exploratory, double blind intervention study with inulin-type fructans in obese women. Gut 62(8).

18. Nakamura Y, Natsume M, Yasuda A, Ishizaka M, Kawahata K, et al. (2017) Fructooligosaccharides suppress high-fat diet-induced fat accumulation in C57BL/6J mice. Biofactors 43(2): 145-151. 
19. Weitkunat K, Schumann S, Petzke KJ, Blaut M, Loh G, et al. (2015) Effects of dietary inulin on bacterial growth, short-chain fatty acid production and hepatic lipid metabolism in gnotobiotic mice. J Nutr Biochem 26(9): 929-937.

20. Ötles S, Ozgoz S (2014) Health effects of dietary fiber. Acta Sci Pol Technol Aliment 13(2): 191-202

21. Rebello CJ, Burton J, Heiman M, Greenway FL (2015) Gastrointestinal microbiome modulator improves glucose tolerance in overweight and obese subjects: A randomized controlled pilot trial. J Diabetes Complicat 29(8): 1272-1276.

22. Johnston KL, Thomas EL, Bell JD, Frost GS, Robertson MD (2010) resistant starch improves insulin sensitivity in metabolic syndrome. Diabet Med 27 (4): 391-397.

23. Fernandes J, Vogt J, Wolever TM (2011) Inulin increases shortterm markers for colonic fermentation similarly in healthy and hyperinsulinaemic humans. Eur J Clin Nutr 65(12): 1279-1286.

24. Delzenne NM, Cani PD, Neyrinck AM (2007) Modulation of glucagonlike peptide 1 and energy metabolism by inulin and oligofructose: experimental data. J Nutr 137(11): 2547-2551.

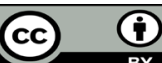

This work is licensed under Creative Commons Attribution 4.0 License

Submission Link: https://biomedres.us/submit-manuscript.php
25. Parnell JA, Reimer RA (2012) Prebiotic fibres dose-dependently increase satiety hormones and alter Bacteroidetes and Firmicutes in lean and obese JCR: LA-cp rats. Br J Nutr 107(4): 601-613.

26. Bonsu KA Nana, Johnson Shanthi (2012) Effects of inulin fibre Supplementation on Serum Glucose and Lipid Concentration in Patients with Type 2 Diabetes. Int J Diabetes \& Metab 21(3): 80-86.

27. Kim Jin Eun, Kim Young Mi, Kim Jin Soo, Cho Kyung Dong, Han Chan Kyu et al. (2011) Effects of Fructooligosaccharides Intake on Body Weight, Lipid Profiles, and Calcium Status among Korean College Students. The FASEB Journal 25(1): 771.

28. Guess ND, Dornhorst A, Oliver N, Bell JD, Thomas EL, et al. (2015) A randomized controlled trial: the effect of inulin on weight management and ectopic fat in subjects with prediabetes. Nutr Metab (Lond) 12: 36.

30. Liu F, Prabhakar M, Ju J, Long H, Zhou HW (2017) Effect of inulin-type fructans on blood lipid profile and glucose level: a systematic review and meta-analysis of randomized controlled trials. European Journal of Clinical Nutrition 71: 9-20.

31. Singh A, Zapata RC, Pezeshki A, Chelikani PK (2017) Dietary lactalbumin and lactoferrin interact with inulin to modulate energy balance in obese rats. Obesity (Silver Spring) 25(6): 1050-1060.

Assets of Publishing with us
BIOMEDICAL
RESEARCHES $\quad \begin{aligned} & \text { Global archiving of articles } \\ & \text { - Immediate, unrestricted online access }\end{aligned}$

